\title{
Les revues syndicales des instituteurs français et l'Éducation nouvelle (1921-1932)
}

\section{Frédéric Mole}

Après la Première Guerre mondiale, les instituteurs syndicalistes français entretiennent avec l'Éducation nouvelle une relation complexe qui se donne à lire en particulier à travers leur presse corporative. Certains acteurs s'impliquent simultanément dans les deux réseaux, à l'instar de Freinet. Au-delà de la figure emblématique de ce grand pédagogue qui développe un mouvement spécifique autour de ses propres techniques, on peut observer diverses formes d'expression des thèmes de l'Éducation nouvelle à l'intérieur du syndicalisme. Idées et pratiques se diffusent au sein des espaces de débats ouverts par les périodiques. En procédant à une comparaison des grandes revues syndicales des années 1920 au début des années 1930 - L'École émancipée (révolutionnaire) et la Revue de l'enseignement primaire qui laisse place à L'École libératrice en 1929 (réformistes) -, l'article analyse le positionnement des deux courants syndicaux vis-à-vis de Freinet et de l'Éducation nouvelle en général (notamment de la revue Pour l'Ère nouvelle).

Après la Première Guerre mondiale, les instituteurs syndicalistes français se rapprochent de l'Éducation nouvelle. Dans un contexte marqué par un élan pacifiste autant que par une espérance révolutionnaire, un renouvellement des ressources pédagogiques leur paraît la condition d'un enseignement adapté aux objectifs sociaux et politiques qu'ils veulent voir assignés à l'école. Sans doute cette préoccupation ne concerne-t-elle pas tous les instituteurs ni même tous les syndiqués, mais au moins une frange de militants que l'on pourrait qualifier de pédagogiques, si le syntagme n'était pas anachronique. Ces acteurs s'expriment surtout dans les périodiques syndicaux, locaux et nationaux, qui prennent une part importante dans ce processus. Cette presse syndicale offre un espace discursif privilégié où se diffusent idées, modèles et auteurs de référence; et où s'ouvrent des débats. Déjà, avant 1914, la Revue de l'enseignement primaire, L'École rénovée puis L'École émancipée avaient initié des débats relevant de problématiques de l'Éducation nouvelle ${ }^{1}$. Après la guerre, les thèmes de l'Éducation nouvelle circulent au sein du syndicalisme. Les syndicalistes cherchent à lier les visées réformatrices en éducation à un projet de transformation de la société. Par sa 
double implication dans le syndicalisme et l'Éducation nouvelle, et surtout par la dynamique qu'il crée autour de ses propres techniques pédagogiques, Célestin Freinet occupe une place éminente dans ce processus. Et s'il n'incarne pas à lui seul la diversité des préoccupations pédagogiques des instituteurs, les syndicalistes sont cependant conduits à prendre en compte l'originalité de sa démarche et de ses inventions, et à préciser leurs propres conceptions d'un enseignement rénové.

Durant cette période, on observe dans ces revues syndicales une modification des conditions du débat. Avant la guerre, les discussions les plus nourries étaient internes à chaque périodique: des militants s'interpelaient et se répondaient d'un numéro à l'autre, comme on le voit tout particulièrement dans L'École émancipée. $\mathrm{Au}$ sein d'organes pourtant liés à des organisations, les rédacteurs jouissaient d'une certaine liberté d'initiative. La ligne éditoriale se dessinait à travers les débats plutôt qu'elle n'y présidait. Dans les années 1920, cette tendance subsiste parfois, mais les débats internes mettent désormais en scène des courants plutôt que des individus. Dans un contexte où les enjeux sociaux et politiques de l'enseignement scolaire sont plus marqués, les controverses impliquent des organes syndicaux et pédagogiques porteurs d'options stratégiques divergentes - réforme ou révolution, pacifisme ou lutte des classes - et engagés dans des organisations internationales ${ }^{2}$.

Cet article examine, dans le foisonnement des démarches et des débats, quelques phénomènes caractéristiques de la place qu'occupent les revues dans l'élaboration des positions pédagogiques des instituteurs syndicalistes et propose une comparaison des analyses de la Fédération unitaire de l'enseignement ${ }^{3}$ (révolutionnaire) et du SNI ${ }^{4}$ (réformiste). La première s'exprime dans L'École émancipée, la seconde dans la Revue de l'enseignement primaire puis dans L'École libératrice à partir de 1929. Malgré certaines convergences de vues, ces deux organisations dessinent deux façons de construire le problème pédagogique et choisissent des démarches éditoriales différentes. Il s'agit donc de mieux comprendre ces deux cultures syndicales et la complexité de leurs positionnements; il s'agit aussi de saisir la façon dont elles se situent vis-à-vis de Freinet dont les positions focalisent l'attention au sein du syndicalisme et dont le rayonnement grandissant fait redouter une tendance à l'hégémonie. À partir de quelques exemples, nous allons donc repérer la façon dont les revues prennent part à l'élaboration et à la circulation des idées pédagogiques, en étant attentif aux références et influences internationales qui s'y expriment, afin de mieux comprendre comment stratégies syndicales et inventivité pédagogique se conjuguent chez ces acteurs. La période étudiée va de 1921 - année marquée par la fondation de la Ligue internationale pour l'Éducation nouvelle (LIEN) - à 1932, année du congrès de Nice de la LIEN, qui constitue un moment de crise dans les rapports de Freinet à l'Éducation nouvelle et où on constate également une variation des positions syndicales à son sujet. Pour en saisir les points saillants, le corpus des revues étudiées fait ici l'objet d'un dépouillement conduit à partir d'une analyse de type qualitatif. 


\section{Syndicalisme et Éducation nouvelle: un intérêt réciproque?}

Déjà engagés avant guerre, notamment par l'implication de Louis Roussel dans la Société libre pour l'étude psychologique de l'enfant d'Alfred Binet (SLEPE) ou par les liens noués par Marius Tortillet (alias M.-T. Laurin) et Gabriel Persigout avec Édouard Claparède 5 , les échanges entre les syndicalistes et les acteurs de l'Éducation nouvelle connaissent de nouveaux développements au début des années 1920. En 1922, Adolphe Ferrière témoigne de son intérêt pour les visées pédagogiques des instituteurs syndicalistes. Cette année-là, la Fédération unitaire de l'enseignement - qui publie la revue L'École émancipée - participe à la création de l'Internationale des travailleurs de l'enseignement (ITE), organisation syndicale marquée par l'influence de la Russie révolutionnaire ${ }^{6}$. L'École émancipée publie peu après une lettre de Ferrière au "secrétaire pédagogique» de cette nouvelle Internationale syndicale et pédagogique. Étrangement, la revue n'évoque pas directement les démarches que l'Internationale aurait faites initialement auprès de Pierre Bovet, directeur de l'Institut Jean-Jacques Rousseau, et de Ferrière, démarches dont cette lettre fait pourtant état. Seule la réponse de Ferrière est publiée par la revue. Celui-ci se montre très attentif à cette prise de contact et l'accueille avec bienveillance. Dans sa réponse, le directeur du Bureau international des écoles nouvelles affirme la possible convergence de vues et d'action entre l'Éducation nouvelle et l'Internationale des travailleurs de l'enseignement. Mettant de côté toute considération idéologique ou politique, il insiste sur l'intérêt d'une collaboration entre les instituteurs syndicalistes français et les instituteurs romands. Cette position est exprimée dans un contexte où les instituteurs romands établissent une collaboration étroite avec l'Institut Jean-Jacques Rousseau: L'Éducateur, organe de la Société pédagogique de la Suisse romande, devient également, de 1921 à 1932, celui de l'Institut Jean-Jacques Rousseau et est alors co-dirigé par Pierre Bovet. Ce périodique intègre également L'Intermédiaire des Éducateurs, bulletin publié par l'Institut. La lettre de Ferrière est représentative d'une période où les acteurs de l'Éducation nouvelle cherchent, notamment par des voies éditoriales, à se rapprocher des enseignants du primaire. Elle mérite d'être amplement citée:

Mon collègue Pierre Bovet m'a communiqué les numéros 1 et 2 du bulletin périodique de l'Internationale de l'Enseignement, et m'a dit que vous seriez désireux d'entrer en rapport avec moi, puisque nous travaillons chacun à la réforme de l'enseignement selon les principes rationnels. J'ai lu avec intérêt ces deux bulletins périodiques. Bien que me plaçant, comme psychologue, en dehors de toute question de parti politique, $j$ 'approuve pleinement les considérations que vous exposez dans la partie pédagogique. Dans le premier bulletin, ce que vous dites de l'enseignement de l'histoire correspond entièrement aux décisions qui ont été prises au $3^{\mathrm{e}}$ Congrès d'éducation morale que vous mentionnez d'ailleurs. [...] J'avais déjà lu, et j'ai relu avec plaisir 
l'article intitulé "L'école du travail», par Innocent Scrishev. Il corrobore entièrement le contenu de mon ouvrage L'école active. [...] En Suisse romande, les instituteurs primaires marchent d'une façon presque unanime à la suite des pionniers de la révolution scolaire. M. Emmanuel Duvillard, président la Société pédagogique de la Suisse romande, est aussi l'auteur d'un ouvrage intitulé Les tendances actuelles de l'école primaire qui est un manifeste en faveur de la réforme rationnelle de l'éducation au sens où vous l'entendez. Je me fais un plaisir de vous envoyer, par le même courrier, le premier numéro de notre revue: Pour L'Ère nouvelle. Si vous le désirez, je pourrais, à l'avenir, vous faire parvenir cette revue en échange du Bulletin périodique de l'Internationale de l'Enseignement, ou mieux encore de L'École émancipée (Ferrière, 1922, p. 209).

Ferrière recourt à une terminologie - «révolution scolaire», «réforme rationnelle», etc. - qui fait manifestement écho aux idéaux politiques syndicalistes. Son discours vise à montrer la possible convergence de mouvements pédagogiques politiquement hétérogènes. En cherchant à prendre en compte les conceptions politiques des destinataires du message, l'argumentaire fait apparaître un certain flottement conceptuel. L'idée de principes rationnels d'enseignement, par exemple, renvoie chez les syndicalistes révolutionnaires français à un cadre d'analyse matérialiste écartant tout spiritualisme. Force est de constater, en revanche, que les "principes de ralliement» de la LIEN comportent une dimension explicitement spiritualiste et n'évoquent pas l'idée d'une pédagogie rationnelle. La rationalité susceptible de réunir tous les éducateurs progressistes reste donc mal définie. En insistant sur la proximité possible entre les instituteurs français et ceux de Suisse romande, Ferrière montre en outre qu'il regarde les associations corporatives d'instituteurs comme l'une des principales ressources dans la perspective d'une réforme scolaire. Pour le fondateur de la LIEN, on doit considérer que les idéaux pédagogiques sont plus puissants que les divergences philosophiques et politiques. Celles-ci ne devraient plus constituer un obstacle, dès lors que l'on s'accorde à fédérer toutes les forces tendant à l'édification d'un système d'enseignement nouveau.

L'École émancipée présente ce texte, dans une rubrique intitulée «Correspondance», de la façon suivante: "Nous croyons utile de publier une lettre qui a été adressée récemment au secrétaire pédagogique de l'Internationale de l'enseignement» (Ferrière, 1922, p. 209). En jugeant opportun de publier la réponse de Ferrière sans faire connaitre explicitement la démarche initiale du secrétaire pédagogique de l'Internationale des travailleurs de l'enseignement, la revue évite d'exprimer l'intérêt que le syndicalisme révolutionnaire lui-même porte aux travaux des penseurs de l'Éducation nouvelle; elle préfere rendre publique la façon dont ceux-ci, en particulier Ferrière, expriment leur sollicitude à l'égard des forces pédagogiques syndicales. C'est là un trait de la position des syndicalistes révolutionnaires. À leurs yeux, les pédagogues de l'Éducation nouvelle ne font bien souvent que théoriser des démarches semblables à celles déjà initiées et mises en œuvre par ailleurs par des instituteurs syndicalistes. Mais en révélant 
cette lettre de Ferrière aux lecteurs, même sans commentaire, la revue témoigne de l'importance que revêt à ses yeux le regard bienveillant porté sur les initiatives pédagogiques des syndicalistes par un éminent représentant de l'Éducation nouvelle.

Dans les revues, certaines rubriques - les chroniques, les correspondances, etc. - ouvrent des espaces où l'on témoigne des rencontres, des échanges, des emprunts et des influences entre les différents acteurs individuels et collectifs engagés dans la perspective d'une réforme scolaire. Sans être ici nécessairement lieux de débat, les revues sont utilisées comme des moyens d'interpellation, des outils rendant visibles des démarches de comparaison et de dialogue entre des mouvements a priori hétérogènes. Elles peuvent constituer des supports au moyen desquels ces mouvements manifestent leurs différences ou au contraire cherchent à rendre visibles leurs stratégies de convergence.

\section{Freinet et Ferrière: L'École émancipée et Pour l'Ėre nouvelle}

L'attitude bienveillante de Ferrière à l'égard des instituteurs syndicalistes est cependant à mettre en regard de la critique que Freinet lui adresse au sujet des expérimentations pédagogiques soviétiques. La question de savoir comment interpréter l'œuvre scolaire de la Russie soviétique occupe une place centrale dans le syndicalisme révolutionnaire.

Avant d'évoquer cette controverse, rappelons que Freinet s'implique à la fois dans le syndicalisme révolutionnaire et dans les congrès de la Ligue internationale pour l'Éducation nouvelle (LIEN). Son but principal est cependant de développer et de promouvoir son propre mouvement spécifique autour des techniques qu'il élabore. Par ses contributions à L'École émancipée, de 1920 jusqu'au début des années 1930, il cherche plus à diffuser les thèmes de l'Éducation nouvelle au sein du syndicalisme révolutionnaire qu'à œuvrer à l'articulation de l'action syndicale collective et de l'Éducation nouvelle. Parallèlement et symétriquement, il cherche à diffuser une conception socialement révolutionnaire de la pédagogie dans les réseaux de l'Éducation nouvelle. Il tend donc à incarner très tôt - et a fortiori rétrospectivement - la figure de l'instituteur syndicaliste révolutionnaire et pédagogue. Mais la place très éminente qui lui est reconnue apparaît aussi, dans l'histoire des relations syndicalisme/pédagogie, très singulière. Et la reconnaissance de cette figure majeure a pu tendre à minorer d'autres formes de développement des idées de l'Éducation nouvelle au sein du syndicalisme. Il s'agit donc à la fois de comprendre les prises de position des deux grands courants syndicaux vis-à-vis de Freinet et d'essayer de repérer chez eux l'expression d'autres démarches réflexives et innovantes.

Pour Freinet, comme pour l'ITE, la Russie soviétique est en passe de réaliser l'utopie de l'Éducation nouvelle à l'échelle d'une nation entière, laquelle se 
donne comme incarnant aux yeux du monde l'idéal d'une humanité libérée. Les libertaires et les communistes critiques sont évidemment beaucoup plus circonspects. Freinet, pour sa part, adhère au modèle russe et considère que même s'il sépare - illusoirement - pédagogie et politique, Ferrière devrait reconnaître l'œuvre pédagogique soviétique.

Maintenant que notre Internationale des travailleurs de l'enseignement est vivante et active, maintenant qu'elle force journaux et revues à s'occuper de la nouvelle éducation russe, il faudra bien que la Ligue Internationale pour l'Éducation nouvelle, que Pour l'Ėre nouvelle emboîtent le pas (Freinet, 1926, p. 215).

Freinet en appelle à la diffusion des expériences soviétiques et compte sur le pouvoir d'interpellation que cette diffusion aurait sur l'évolution des débats pédagogiques au sein des journaux et revues occidentales. Freinet, qui témoignera toute sa vie d'un grand respect pour Ferrière, tant pour sa personne que pour son œuvre, ne peut se résoudre à le voir réservé ou silencieux sur la question russe: "Je m'attendais à mieux. Je me disais que M. Ferrière, ce pionnier de l'Éducation nouvelle, serait toujours à l'avant-garde, réfléchi, mais ferme [...] et qu'il nous montrerait la voie en nous aidant à nous garer prudemment des dangers. Il s'est tu !» (Freinet, 1926, p. 215). Convaincu d'une synthèse possible, au plan international, entre révolution socialiste et révolution pédagogique, Freinet adopte une ligne de conduite complexe à l'égard de celui qui restera toujours à ses yeux l'une des figures les plus respectables de l'Éducation nouvelle européenne. S'il lui adresse les critiques les plus fermes et les plus nettes, il ne veut jamais aller jusqu'au point de rupture avec l'homme et ses principes et réaffirme toujours avec vigueur sa reconnaissance de l'œuvre pédagogique accomplie par celui qu'il nomme le mâ̂tre: «Nous tâcherons de profiter de sa grande expérience; nous relirons toujours avec profit et gratitude les beaux ouvrages qu'il a écrits sur l'éducation; nous suivrons avec plus d'attention que jamais l'effort de la Ligue internationale pour l'Éducation nouvelle et du Bureau international des écoles nouvelles.» Mais si Ferrière pose les justes principes de l'Éducation nouvelle, son spiritualisme l'empêche d'en apercevoir les conséquences politiques ultimes: Freinet se voit ainsi, à partir du mouvement dont il commence à organiser le déploiement, comme le seul théoricien du point de convergence entre Education nouvelle et révolution, le seul acteur de leur possible synthèse.

Pour Freinet, l'une des fonctions de la presse pédagogique révolutionnaire dans le champ pédagogique réside dans sa fonction d'exhortation, de mise en demeure: montrer aux instituteurs que cette presse doit, si elle veut jouer pleinement son rôle d'avant-garde, mettre tout en œuvre pour rendre possible une convergence entre le mouvement de l'Éducation nouvelle occidentale et la révolution scolaire soviétique. Fidélité à l'Éducation nouvelle, donc, mais volonté résolue d'en surmonter les impasses, ainsi se dessine la position dialectique et la stratégie éditoriale de Freinet dans L'École émancipée: «Il y a un bout de chemin 
qu'il faut nous résoudre à faire seuls désormais, celui qui mène de la pédagogie bourgeoise à la pédagogie révolutionnaire " (Freinet, 1926, p. 215).

Les publications de Freinet durant cette période traduisent son implication assumée dans plusieurs réseaux. Sa ligne de conduite est délicate. Il dénonce les contradictions de l'Éducation nouvelle bourgeoise, son incapacité à prendre en compte la question sociale; mais il explique dans le même temps qu'un point de ralliement est toujours potentiellement possible avec l'Éducation nouvelle soviétique. Ce pari d'une synthèse possible montre l'originalité de la position de Freinet, dans un milieu politique où la critique des promoteurs dits «bourgeois» de l'Éducation nouvelle est fréquente. Durant cette période où il publie de nombreux articles visant à faire connaître les grandes figures de la pédagogie moderne aux lecteurs de L'École émancipée, on peut lire d'autres analyses au sein de la même revue. Par comparaison, elles font mieux comprendre l'originalité de la position de Freinet. La direction de la revue, qui ne souhaite pas paraittre approuver la proximité de Freinet avec des pédagogues jugés «bourgeois», accorde une tribune à ceux qui dénoncent les compromissions de l'Éducation nouvelle avec un régime économique d'exploitation. La revue cite ainsi J. C. Ceton, instituteur hollandais, qui écrit:

Beaucoup de pédagogues bourgeois ont développé de belles théories sur l'éducation. [...] Pour une certaine part, c'était des rêves utopiques, des fantaisies sonores qui n'étaient susceptibles d'aucune application, parce que l'éducation des masses est complètement dépendante du régime social. Le but était toujours de rendre la classe exploitée aussi habile que possible dans la production et d'autre part de maintenir cette classe dans la plus grande sujétion (Ceton, 1923, p. 446).

Autrement dit, l'Éducation nouvelle occidentale est, au mieux, inutile, au pire un nouvel instrument de domination. Même la Revue de l'enseignement primaire, réformiste, bientôt poussée par Georges Lapierre à se rapprocher des grandes innovations pédagogiques, estime opportun, en 1923, de citer un article d'Alix Guillain, extrait de L'Humanité, hostile à Maria Montessori et plus généralement à l'Éducation nouvelle:

Hélas, les pédagogues sont pour la plupart encore des monarques, et même s'ils voulaient s'inspirer des méthodes de Maria Montessori, on se demande, non sans une certaine tristesse, à quoi cela aboutirait s'ils les appliquaient dans une société bourgeoise telle que la France. Que peut-il servir aux enfants d'apprendre à se développer librement et à s'entraider si la vie plus tard les force à faire le contraire, s'il faut étant grands qu'ils se soumettent à un patron brutal, et que la loi de concurrence universelle les force, bon gré mal gré, à considérer leur voisin comme un rival. Le danger de toutes ces méthodes pédagogiques nouvelles sera toujours de ne pas tenir suffisamment compte des rapports étroits qui existent entre un ordre économique donné et ses différentes institutions tant politiques que sociales telles que le gouvernement, la justice, l'instruction publique, etc. (Guillain, 1923, p. 299). 
En somme, toute forme d'Éducation nouvelle mise en œuvre en régime «bourgeois» serait vouée à l'échec. Alors que la presse révolutionnaire jette parfois le discrédit sur les idées éducatives nouvelles de l'Europe occidentale au motif qu'elles seraient inapplicables ou porteuses d'effets pervers, Freinet se refuse systématiquement à ce défaitisme pédagogique. Même s'il est convaincu que seule une révolution sociale offrirait les conditions d'une véritable éducation émancipatrice, les techniques nouvelles lui paraissent devoir être expérimentées et mises en œuvre dans une société encore traversée par des contradictions. Car il fait le pari qu'elles peuvent y produire de profonds effets de transformation sociale.

\section{Eugène Delaunay, un passeur}

L'attitude de Ferrière concernant la pédagogie soviétique est l'occasion d'un désaccord très révélateur des enjeux stratégiques qui animent alors les politiques éditoriales. Ce désaccord oppose Freinet et Eugène Delaunay sur la question de savoir si Ferrière accorde une place suffisante au traitement de la politique scolaire et des expériences pédagogiques soviétiques dans Pour l'Ére nouvelle. Il nous faut tout d'abord évoquer la figure encore trop méconnue d'Eugène Delaunay, instituteur du Calvados, qui joue un rôle important durant cette période dans la circulation des idées entre Éducation nouvelle et syndicalisme. Delaunay écrit dans de nombreuses revues de l'époque, syndicales ou pédagogiques. On trouve sa signature notamment dans L'École émancipée mais aussi dans la Revue de l'enseignement primaire, puis dans L'École libératrice. Il contribue également à La Révolution prolétarienne, revue syndicaliste révolutionnaire dirigée par Pierre Monatte. Dans toutes ces revues, il aborde les questions de l'Éducation nouvelle. Mais symétriquement, il donne aussi une visibilité aux activités pédagogiques émanant du syndicalisme dans Pour l'Ére nouvelle. Car c'est cet instituteur que Ferrière choisit pour tenir la rubrique Chronique française dans la revue de la LIEN à partir de 1926, rubrique dont il assure la rédaction jusqu'en 1939.

Delaunay fait circuler les idées en les adaptant au contexte d'énonciation déterminé par le lectorat des divers périodiques; il joue un rôle de passeur. Dans sa très informée Chronique française, il rend compte régulièrement des activités pédagogiques de Freinet et lui apporte un soutien constant. Mais il replace l'essor du mouvement impulsé par Freinet dans un environnement beaucoup plus large, intégrant d'autres acteurs, d'autres sources. Dans le syndicalisme, le mouvement de réforme en éducation ne se résume pas à l'œuvre de Freinet, il s'ouvre à une pluralité de ressources nouvelles. Et si le désaccord sur la question russe nous intéresse ici, c'est qu'il est révélateur d'enjeux éditoriaux. Sans entrer ici dans le détail de la controverse, il faut en rappeler les conditions au regard de la politique des revues. Lorsque Freinet se plaint du peu d'empressement de Ferrière à rendre compte des expériences pédagogiques soviétiques et qu'il estime 
que c'est seulement l'internationalisme syndical qui peut contraindre Ferrière à prendre en compte l'expérience russe, le syndicaliste Delaunay (1926a, p. 291) défend le directeur de la revue de la LIEN: "Où voit-on que l'Internationale des travailleurs de l'enseignement a forcé Ferrière 'à s'occuper de la nouvelle éducation russe' ?». Mais surtout, il ajoute cette remarque d'ordre très stratégique: «Ne devine-t-on pas au contraire que de telles affirmations ne sont pas faites pour nous ouvrir les colonnes des journaux et des revues ?»

La réponse de Delaunay se situe donc à un double niveau. D'une part, il conteste que Pour l'Ėre nouvelle ait négligé de s'intéresser aux expérimentations russes; mais il juge surtout, d'autre part, cette attaque de Freinet contraire à l'esprit d'ouverture dont il se veut, par sa position, le garant. Ferrière aurait légitimement temporisé et attendu d'être mieux documenté: avant de faire connaître l'expérience pédagogique russe, «il nous faut la connaître nous-mêmes» explique Delaunay dans L'École émancipée (1925, p. 161). Quelques mois plus tard, Delaunay consacre deux articles à la question dans Pour l'Ére nouvelle en précisant en introduction qu'il entend, sur cette question, se tenir à distance «des adversaires sans scrupules» comme «des amis trop enthousiastes» (1926b, p. 77). Dans cette affaire, la démarche de Delaunay témoigne d'une cohérence qui s'observe dans toutes ses contributions: montrer la compatibilité entre toutes les tendances visant la transformation de l'enseignement scolaire, qu'elles proviennent de théoriciens genevois ou de praticiens révolutionnaires. Sa défense de Pour l'Ére nouvelle dans les colonnes mêmes de L'École émancipée peut-être comprise comme la contrepartie logique de l'esprit d'ouverture qui prévaut dans sa rubrique "Chronique française» dans Pour l'Ëre nouvelle.

Bien qu'ayant en commun cette singularité d'être impliqué dans les deux mouvements (syndicalisme et Éducation nouvelle) et d'être en accord au plan pédagogique, Freinet et Delaunay adoptent des stratégies divergentes. Le premier, pédagogue militant, assume une position de franc-tireur dans le débat public et cherche à convaincre, avec une grande assurance, de la prochaine suprématie d'une pédagogie révolutionnaire dont l'avant-garde se trouve en URSS. Le second, chroniqueur subtil et nuancé, est soucieux de préserver une ouverture à la diversité des analyses et des pratiques. Ainsi se dessinent deux attitudes éditoriales qui illustrent les tensions que vivent les instituteurs syndicalistes face aux revendications d'une Éducation nouvelle qui cherche alors son sens à l'échelle mondiale.

\section{Révolutionnaires et réformistes face à Freinet}

C'est un fait bien établi: Freinet est écarté de la rédaction de L'École émancipée au début des années $1930^{7}$. La direction de la revue syndicaliste révolutionnaire rejette sa propension à revendiquer une position hégémonique et se prononce pour un "pluralisme pédagogique» au sein de la revue (Robert, 2006, 
p. 23). Ce qui nous intéresse ici, c'est un renversement spectaculaire des conditions de visibilité des idées pédagogiques de Freinet à travers les deux grandes revues syndicales. Bien que demeurant impliqué dans le camp révolutionnaire, notamment par ses responsabilités à l'Internationale des travailleurs de l'enseignement, Freinet se tourne plutôt vers L'École libératrice à partir du congrès de Nice de la LIEN, en 1932.

Le contexte du congrès de Nice exacerbe en effet les tensions ${ }^{8}$. Les divergences de perception de l'œuvre et de l'action de Freinet s'accentuent fortement entre L'École émancipée et L'École libératrice. Gabrielle Bouët (1932, pp. 77-78) dénonce dans L'École émancipée «'hypocrisie de ces assises ultra-bourgeoises où l'on fait apparemment de beaux exposés sur la liberté de l'enfant, 'l'Éducation dans ses rapports avec l'évolution sociale', mais où l'on reste nationalement sur ses positions, légitimant [...] les mesures les plus réactionnaires contre l'école populaire». Le Congrès de Nice est donc discrédité, au point que Gabrielle Bouët n'évoque à aucun moment la présence de Freinet, ni bien sûr l'exposition qu'il y présente sur L'Imprimerie à l'école:

Ce qui nous paraît fâcheux, explique-t-elle, c'est l'engouement de certains collègues, voire de camarades pour ces démonstrations officielles et tapageuses [...]. Nous pensons que les syndicats ne doivent pas se laisser déposséder ainsi d'une de leur principale raison d'être: l'amélioration de notre technique. [...] Nous devons être des entraîneurs, non des suiveurs (Bouët, 1932, p. 77-78).

Cette position marque la rupture avec Freinet, accusé implicitement d'une inacceptable compromission. Elle repose donc sur une double affirmation. D'une part, les instituteurs syndicalistes doivent être seuls à l'initiative des inventions pédagogiques. D'autre part les manifestations de la pensée pédagogique dite «bourgeoise» ne peuvent constituer qu'un obstacle à l'initiative des instituteurs.

Dans L'École libératrice, l'analyse est diamétralement opposée. J. Flaesch, institutrice à Nice, y rend compte du Congrès de façon très positive. Alors que L'École émancipée dénonce les luxueuses conditions d'organisation du Congrès, en particulier du congrès Montessori organisé au sein du Congrès de la LIEN - comme Élise Freinet d'ailleurs, qui ironise sur les élèves «singes savants» de la pédagogue italienne -, J. Flaesch n'en restitue la présence qu'en déclarant avoir «beaucoup admiré le calme et la contention d'esprit de ces petits garçons et de ces petites filles». L'École libératrice fait en outre l'éloge de l'exposition de Freinet sur L'imprimerie à l'école - «vif succès» - et souligne que de nombreux congressistes sont allés voir son école, à Saint Paul de Vence» (Flaesch, 1932, pp. 135-136).

Certes, les préoccupations pédagogiques des instituteurs de L'École libératrice ne se situent pas dans la perspective révolutionnaire d'un Freinet. Il s'agit moins pour eux de réinventer les techniques fondamentales de l'école que de réunir les conditions permettant à l'élève de sortir de la passivité dans laquelle il est maintenu ordinairement, de développer une pédagogie active dans le cadre 
institutionnel. L'esprit de réforme doit d'abord inciter les militants à conduire une analyse des programmes et des pratiques. Pourtant, le choix de soutenir Freinet, et même de lui accorder une place dans les pages de la revue, s'explique de la façon suivante. Georges Lapierre, figure centrale du SNI, est secrétaire de rédaction de la revue. Il cherche à réunir toutes les conditions d'une rencontre entre un syndicalisme réformiste et les idées pédagogiques nouvelles, tant au plan national qu'international. Or, sur ce plan, Freinet est devenu incontournable. La rentrée 1932 est l'occasion d'un nette accentuation des ambitions du SNI au plan pédagogique. Elle se fait sous le signe des congrès qui marquent l'essor des idées nouvelles dans l'enseignement. Dans son éditorial “'École libératrice” 1932» - dont le titre même exprime l'intention d'un nouveau départ -, Georges Lapierre détaille le mouvement général dans lequel il voit s'inscrire désormais l'œuvre pédagogique des syndicalistes. Il situe le congrès du SNI et ses activités dans le contexte des grands congrès internationaux. Il insiste en particulier sur les fortes convergences entre le syndicalisme et les visées du congrès de Nice de la LIEN. Il insiste également sur l'intérêt des techniques nouvelles: l'«Imprimerie à l'école», les "projections, radio, disques", ces techniques "retiendront notre attention par la contribution qu'elles apportent au renouvellement des méthodes pédagogiques», explique-t-il (Lapierre, 1932, p. 3).

Au cours de cette année 1932 - où Freinet durcit pourtant ses positions et donne un nouveau titre à sa revue (L'Éducateur prolétarien remplace L'Imprimerie à l'école) -, Lapierre décide que l'organe du SNI sera plus accueillant au pédagogue révolutionnaire. Alors que c'est Freinet lui-même qui refusait de publier quelques années auparavant dans la Revue de l'enseignement primaire ${ }^{9}$, on se réjouit désormais, au sein de la revue de Freinet, de ces nouvelles perspectives éditoriales:

L'Imprimerie à l'École a obtenu droit de cité dans L'École Libératrice. Lapierre a fait savoir à Alziary et a confirmé au Congrès de Clermont que quatre pages seraient cette année réservées à notre technique. [...] C'est la preuve que l'Imprimerie à l'École, grâce à son développement continu et à ses qualités particulières, s'est imposée aux éducateurs comme une technique novatrice et ne peut continuer à être négligée par une revue qui s'adresse à la majorité du personnel. Le directeur de L'École Libératrice avait même songé un moment à lui consacrer une rubrique permanente (Lelache, 1932, pp. 17-18).

Saisissant l'occasion de ce «brusque changement d'attitude» (Lelache, 1932, p. 18), Freinet décide, avec des camarades de son mouvement impliqués dans le Syndicat national des Instituteurs, de soumettre au congrès du syndicat une motion relative à la création d'une rubrique qui leur serait réservée dans $L$ 'École Libératrice:

Faisant droit à la légitime réclamation des instituteurs syndiqués qui, par la recherche et la mise en ouvre de techniques nouvelles, s'efforcent de mettre l'enseignement en harmonie avec les besoins et les possibilités de la nature 
enfantine et, d'autre part, avec les conceptions syndicales de la vie sociale; Considérant que L'Imprimerie à l'École est expérimentée dans des centaines de classes et qu'elle apparait, à ses adeptes, comme une technique rénovatrice et libératrice qui introduit à l'école deux éléments essentiels, la liberté et l'effort collectif, qui manquent à notre enseignement officiel; que, par suite, elle mérite d'être connue et a droit à l'attention bienveillante et au soutien du SN ${ }^{10}$ :

Décide l'institution d'une rubrique permanente consacrée aux expérimentations et aux applications des techniques nouvelles d'enseignement, en premier lieu L'Imprimerie à l'École, la direction et la tenue de cette rubrique devant être réservées aux maîtres syndiqués compétents (Lelache, 1932, 18).

Le simple fait qu'une telle proposition ait pu être soumise à un congrès syndical indique que le syndicalisme réformiste s'ouvre alors à une pluralité d'influences pédagogiques, qu'il devient envisageable que la démarche et les techniques d'un grand pédagogue dont l'influence s'avère grandissante soient reconnues comme telles par le syndicat et qu'une place leur soit accordée dans son organe de presse. Mais le fait que la question n'ait pas été mise en discussion indique aussi que cette idée - un moment envisagée par Lapierre lui-même - ne s'avère pas encore recevable dans les instances de décision du syndicat.

Cet esprit d'ouverture et d'engagement ne concerne pas Freinet exclusivement, mais l'Éducation nouvelle en général. En novembre 1932, J. Flaesch résume la ligne éditoriale impulsée par Lapierre pour L'École libératrice. Elle consiste à promouvoir auprès des syndiqués et des instances syndicales ellesmêmes un très net rapprochement avec la Ligue internationale pour l'Éducation nouvelle. «Puisque la Ligue d'Éducation nouvelle et le Syndicalisme poursuivent en partie le même objet, pourquoi ne s'associeraient-ils pas sur le point qui leur est commun ?» demande J. Flaesch. Elle résume l'enseignement qui lui paraît se dégager du Congrès de la façon suivante: $1 /$ «Le plus grand nombre possible de syndicalistes devraient adhérer au groupe français d'Éducation nouvelle» (GFEN); 2/ "Chaque section du SN devrait y adhérer aussi»; 3/ "Chaque section syndicale devrait constituer des groupes locaux de recherches et d'efforts communs» (Flaesch, 1932b).

\section{Conclusion}

Du début des années 1920 au début des années 1930, les instituteurs syndicalistes sont confrontés à l'essor de modèles pédagogiques nouveaux, que ces modèles aient élaborés en leur propre sein par des pédagogues inventifs au premier rang desquels se trouve Freinet, ou qu'ils aient été élaborés par des penseurs n'appartenant ni au syndicalisme et ni même au corps des instituteurs, mais au mouvement de l'Éducation nouvelle. On observe que les militants de la revue 
L'École émancipée ont d'abord témoigné d'un esprit d'ouverture en direction de Ferrière et de l'Éducation nouvelle et ont largement ouvert les pages de la revue syndicaliste au jeune Freinet, avant de se montrer jaloux de leur prérogatives et de tendre à se replier, au plan pédagogique, sur une forme d'esprit de corps qu'ils entendaient cantonner dans une certaine orientation révolutionnaire. Dans le même temps, L'École libératrice adoptait, sous l'impulsion de Lapierre, une ligne diamétralement opposée, recherchant dans diverses directions à relayer les idées nouvelles et à s'associer à différents réseaux d'acteurs. Une "Chronique d'éducation nouvelle» est créée en 1931. Il est particulièrement caractéristique qu’à l'occasion de la parution du livre de Pierre Bovet, Vingt ans de vie. L'Institut J.-J. Rousseau de 1912 à 1932, on écrive dans L'École libératrice: "À quand, en France, la création d'un Institut supérieur de pédagogie ? «La réforme de l'enseignement serait incomplète sans lui» (Duthil, 1932). L'urgence d'une innovation pédagogique trouve alors dans les revues corporatives des instituteurs français une expression grandissante. Celles-ci offrent un lieu de transfert où les idées éducatives nouvelles d'origines diverses sont discutées et intégrées à un projet syndical et politique de transformation de la société.

\section{Notes}

1 Voir nos travaux: (2010). L'école läque pour une République sociale. Controverses pédagogiques et politiques (1900-1914). Rennes: PUR / Lyon: INRP, 2e partie; (2011). L'école rénovée: une revue d'éducation nouvelle entre anarchisme et syndicalisme (1908-1909)», in «Histoire du mouvement de l'éducation nouvelle» (Laurent Gutierrez, coord.), Carrefours de l'éducation, 31, 9-22.

2 La Fédération internationale des Associations d'instituteurs pour le courant réformiste à partir de 1926, l'Internationale des travailleurs de l'enseignement pour le courant révolutionnaire à partir de 1922.

3 Voir Le Bars Loïc (2005). La Fédération unitaire de l'enseignement (1919-1935). Paris: Syllepse. Le nom officiel de la fédération est: Fédération des syndicats des membres de l'enseignement laïque.

4 Le sigle du Syndicat national des instituteur est durant cette période: SN. Pour plus de lisibilité, c'est SNI qui est utilisé ici, comme c'est l'usage quelques années plus tard.

5 Voir note 1.

6 Voir Laurent Frajerman (2001). Le rôle de l'Internationale des Travailleurs de l'Enseignement dans l'émergence de l'identité communiste enseignante en France (1919-1932). Cahiers d'histoire. Revue d'histoire critique, 85, 111-126.

7 Sur ce conflit entre Freinet, L'École émancipée et la Fédération unitaire de l'enseignement, voir André D. Robert (2006). Miroir du syndicalisme enseignant. Paris: Nouveaux regards/ Syllepse, chap. 1.

8 Voir notre étude «Freinet au Congrès mondial de Nice (1932): une révolution sociale par l'Éducation nouvelle ?», in Laurent Gutierrez, Laurent Besse \& Antoine Prost (Éd.), Réformer l'école. L'apport de l'Éducation nouvelle (1930-1970) Grenoble: PUG, p. 63-75.

9 Voir à ce sujet Le Bars Loïc (2005). La Fédération unitaire de l'enseignement (1919-1935). Paris: Syllepse, p. 218.

10 Voir note 4. 


\section{Bibliographie}

Bouët Gabrielle (1932, 30 octobre). À propos du Congrès de Nice. L'École émancipée, 77-78.

Ceton J. C. (1923, 5 mai). L'école communiste - Le but de l'éducation. L'École émancipée, 446-447.

Delaunay Eugène (1925, 13 décembre). Pédagogie russe et pédagogie bourgeoise. L'École émancipée, 12, 160-161.

Delaunay Eugène (1926a, 14 février). Pédagogie russe et pédagogie bourgeoise. L'École émancipée, 21, 291.

Delaunay Eugène (1926b, juillet). L'effort pédagogique russe (I). Pour l'Ẻre nouvelle, 21, 77-82.

Duthil, R. (1932, 19 novembre). Pour la bibliothèque du travail du maître. Vingt ans de vie. L'Institut J.-J. Rousseau de 1912 à 1932, par Pierre Bovet. L'École libératrice, 8, 232.

Ferrière Adolphe, directeur du Bureau international des écoles nouvelles, à Genève (1922, 30 décembre). Lettre au secrétaire pédagogique de l'Internationale de l'enseignement. L'École émancipée, 209.

Flaesch J. (1932a, 29 octobre). VI e Congrès mondial d'Éducation nouvelle. L'École libératrice, 5, 135-136.

Flaesch J. (1932b, 12 novembre). Au Congrès d'Éducation nouvelle de Nice. L'École libératrice, $7,185$.

Frajerman Laurent (2001). Le rôle de l'Internationale des Travailleurs de l'Enseignement dans l'émergence de l'identité communiste enseignante en France (1919-1932). Cahiers d'histoire. Revue d'histoire critique, 85, 111-126.

Freinet Célestin (1926, 3 janvier). Pédagogie russe et pédagogie bourgeoise. L'École émancipée, 231-215.

Guillain Alix (1923, 9 septembre). La méthode Montessori, Revue de l'enseignement primaire, 298-299 (article d'abord paru dans L'Humanité).

Lapierre Georges (1932, octobre). «École libératrice» 1932. L'École libératrice, 1, 2-3.

Le Bars Loïc (2005). La Fédération unitaire de l'enseignement (1919-1935). Paris: Syllepse.

Lelache, S. (1932, octobre). L'Imprimerie à l'école et L'École libératrice. L'Éducateur prolétarien, 1, 17-18.

Mole Frédéric (2010). L'école laïque pour une République sociale. Controverses pédagogiques et politiques (1900-1914). Rennes: PUR / Lyon: INRP.

Mole Frédéric (2012). Freinet au Congrès mondial de Nice (1932): une révolution sociale par l'Éducation nouvelle? In Laurent Gutierrez, Laurent Besse \& Antoine Prost (Éd.), Réformer l'école. L'apport de l'Éducation nouvelle (1930-1970) Grenoble: PUG, p. 63-75.

Robert André D. (2006). Miroirs du syndicalisme enseignant. Paris: Nouveaux regards/Syllepse.

Mots-clés: Instituteurs syndicalistes, Éducation nouvelle, Célestin Freinet, réforme pédagogique 


\section{Gewerkschaftliche französische Lehrerzeitschriften und die Reformpädagogik (1921-1932)}

\section{Zusammenfassung}

Nach dem Ersten Weltkrieg unterhalten die gewerkschaftlich organisierten französischen Lehrerinnen und Lehrer komplexe Beziehungen zur Reformpädagogik, wie sich an ihren Zeitschriften ablesen lässt. Gewisse Vertreter engagieren sich in beiden Netzwerken, so wie es Freinet tut. Neben der emblematischen Figur dieses grossen Pädagogen, der seine eigene Gefolgschaft hat, lassen sich verschiedene andere Arten des Umgangs mit der Reformpädagogik beobachten. Indem zwei wichtige gewerkschaftliche Zeitschriften, die 〈revolutionäre` L'École émancipée und die «reformierte` Revue de l'enseignement primaire, verglichen werden, kann der Beitrag die Standpunkte der beiden gewerkschaftlichen Strömungen gegenüber Freinet und der Reformpädagogik (besonders wie sie in Pour l'Ėre nouvelle vertreten wurde) untersuchen.

Schlagworte: Gewerkschaftlich organisierte Lehrerinnen und Lehrer, Reformpädagogik, Célestin Freinet, Schulreform

\section{Riviste sindacali dei maestri francesi e la Nuova Educazione (1921-1932)}

Riassunto

Dopo la Prima Guerra mondiale, i maestri sindacalisti francesi intrattengono con la Educazione Nuova une relazione complessa, che traspare particolarmente dalla stampa corporativa. Alcuni attori, come Freinet, si implicano simultaneamente nelle due reti. Oltre alla figura emblematica di questo grande pedagogo, che sviluppa un movimento specifico attorno alle proprie tecniche, si possono osservare diverse forme d'espressione dei temi promossi dalla Nuova Educazione all'interno del movimento sindacale. Idee e pratiche si diffondono in seno agli spazi di dibattito aperti da questi periodici. Procedendo a una comparazione delle grandi riviste sindacali dagli anni '20 all'inizio degli anni ' 30 - L'École émancipée (di stampo rivoluzionario) e la Revue de l'enseignement primaire, che lascerà poi spazio a L'École libératrice nel 1929 (entrambe riformista) - l'articolo analizza il posizionamento delle due correnti sindacali nei confronti di Freinet e della Nuova Educazione in generale (specialmente per quanto riguardo la rivista Pour l'Ère nouvelle).

Parole chiave: Maestri sindacalisti, Nuova Educazione, Célestin Freinet, riforma educativa 


\section{The union journals of the French teachers and the New Education (1921-1932)}

\section{Summary}

After World War I, the French union teachers had a complex relationship with the New Education movement; this can be read in particular in their corporate media. Some actors were involved simultaneously in both networks, like Freinet. Beyond the emblematic figure of this great teacher who developed a specific movement around his own techniques, one can observe various forms of expression of the New Education's themes within the unionism. Ideas and practices did spread in areas of debates such as periodicals. Comparing major union journals from the 1920s to the early 1930s - L'École émancipée (revolutionary) and Revue de l'Enseignement primaire which leaves room to L'École liberratrice in 1929 (reformist) - the article analyzes the positioning of both union currents towards Freinet and the New Education in general (including the Pour l'Ere Nouvelle journal).

Keywords: Union teachers, New Education, Célestin Freinet, pedagogical reform 